\title{
Perception paysanne de l'utilisation de Diospyros mespiliformis Hochst. ex A. Rich Au Niger.
}

\author{
Ado ALI ${ }^{1 *}$, Manirou OUMAROU ${ }^{2}$ Soumaila MOUNKAILA ${ }^{1}$, Ali MAHAMANE ${ }^{2}$, Mahamane \\ SAADOU ${ }^{2}$ \\ ${ }^{1}$ Université d'Agadez, Faculté des Sciences et Techniques; BP 199 Agadez; Niger \\ ${ }^{2}$ Université Abdou Moumouni de Niamey, Faculté des Sciences et Techniques, Département de Biologie \\ Laboratoire Garba Mounkaila, BP 10662 Niamey, Niger \\ *Correspondant: Ado ALI, Université d'Agadez, Faculté des Sciences et Techniques; BP 199 Agadez; \\ Niger. Tel: +227-9890-0215. E-mail: aaliadok@gmail.com
}

Submitted on $8^{\text {th }}$ March 2021. Published online at www.m.elewa.org/journals/ on 30th April 2021 https://doi.org/10.35759/JABs.160.3

\section{RÉSUMÉ}

Objectif: Diospyros mespiliformis est une espèce fruitière très appréciée par la population rurale. Cette étude vise à caractériser l'impact son utilisation au Niger principalement dans les régions de Dosso, Maradi et Tillabéri.

Méthodologie et Résultats : Une enquête ethnobotanique était réalisée en utilisant un échantillonnage stratifié à trois niveaux (phytogéographique, hydrographique et administratif) et portant sur 600 répondants. Les résultats ont montré que c'est une plante sauvage intensément exploitée dont la distribution spatiale est fonction de zones agro-climatiques. Son délai de première production fruitière varie entre 5 et 11 ans selon $41,25 \%$ des enquêtés. En effet, toutes ses parties sont utilisées ce qui contribue à sa régression (98,2\% de répondants). Ainsi, les fruits sont vendus localement entre 100 et $400 \mathrm{~F}$ cfa le kilogramme et le bois commercialisé. Ses parties traitent plus de 33 symptômes et maladies.

Conclusions et application de la recherche: Diospyros mespiliformis est une espèce en régression du fait de sa surexploitation au Niger. En dépit des fonctions et des services fournis, $D$. mespiliformis ne fait pas l'objet d'une protection particulière même si les ruraux tentent tant bien que mal de le faire. Ce travail contribue à une prise de décision pour freiner l'érosion génétique de cette espèce.

Mots clés : Diospyros mespiliformis, utilisations, ruraux, Niger.

\section{Peasant's perception of Diospyros mespiliformis Hochst. ex A. Rich use of in Niger.}

\begin{abstract}
Objective: D. mespilifomis is a prized species among the peasants. This study aims to characterize its use in Dosso, Maradi and Tillabéri areas in Niger country.

Methodology and Results: An ethnobotanical survey was carried out using a stratified sampling at three levels (phytogeographic, hydrographic and administrative) and including 600 persons. The results showed that it is an intensely exploited wild plant whose spatial distribution is a function of agro-climatic zones. Its first fruit production varies between 5 and 11 years
\end{abstract}


according to $41.25 \%$ of respondents. Indeed, all its parts are used which contributes to its regression (98.2\% of respondents). Thus the fruits are sold locally between 100 and 400F cfa per kilogram and the timber traded. Its parts treat more than 33 symptoms and diseases.

Conclusions and application of findings: Diospyros mespiliformis is a regressed species beacause of its anthropogenic use in Niger. Despite these functions and services provided, D. mespiliformis is not the object of special protection even if the rural ones try somehow to do so. This study contributes to a decision making to slow down genetic erosion of this species.

Keywords: Diospyros mespiliformis, uses, rural, Niger.

\section{INTRODUCTION}

En Afrique, les populations locales sont extrêmement dépendantes des produits issus de la végétation spontanée comme les PFNL (produits forestiers non ligneux) qui constituent une principale source de revenus et de compléments alimentaires surtout en zone rurale. Aussi, les actions thérapeutiques d'une gamme assez variée de plantes, bien que non scientifiquement confirmées, ont été découvertes par les peuples depuis des siècles (Hutchings et al., 1996). On dénombre de nombreux ligneux des forêts tropicales dont toutes les parties ou presque sont quotidiennement utilisées en médecine traditionnelle mais aussi comme bois d'œuvre ou bois de service (Fandohan et al., 2008). Ainsi, il existe plusieurs exemples d'essences ligneuses menacées du fait d'une surexploitation (Sinsin et al., 2004 ; Akpona et al., 2009). C'est le cas de D. mespiliformis (Saadou, 2007 ; Ali et al., 2016) dont aucune partie n'échappe à des usages domestiques (Arbonnier, 2000). L'étude de la structure en

\section{MATÉRIEL ET MÉTHODES}

Zone d'étude : L'étude a été réalisée dans les régions de Maradi, Dosso et Tillabéri. La région de Maradi est située entre $6^{\circ}$ et $9^{\circ}$ de longitude Est ; $13^{\circ}$ et $16^{\circ}$ de latitude Nord, en climat sahélien (la pluviométrie est comprise entre 200 et $600 \mathrm{~mm}$ par an). La végétation est composée de forêts basses sèches, forêtgaleries, savanes et steppes (Mahamane et al., 2009). La population est 3402094 habitants soit $49,19 \%$ d'hommes et 50,81 de femmes avec une densité de $81,39 \mathrm{hts} / \mathrm{Km}^{2}$ (INS, 2014). Les régions de Dosso et de Tillabéri se diamètre et en hauteur de $D$. mespiliformis révèle une instabilité de sa population émanant des surexploitations et faible régénération naturelle (Ali et $a l ., 2017)$. En effet, la mise en œuvre de stratégies globales de conservation, de restauration en milieu naturel et de production compatibles avec le développement local de cette plante s'avère nécessaire pour assurer sa pérennité. Cela n'est réaliste que s'il repose sur une base scientifique bien documentée. L'acquisition de données fiables actualisées sur les différents organes utilisés, les considérations socioculturelles, l'ethnobotanique de l'espèce et les pratiques néfastes sur la population de D. mespiliformis est donc d'une impérieuse nécessité pour une meilleure gestion de la ressource. L'objectif visé par cette étude est d'évaluer le niveau de pression qui pèse sur $D$. mespiliformis à travers les différentes formes de son utilisation, afin d'une meilleure gestion de cette ressource.

situent entre $11^{\circ}$ et $16^{\circ}$ de longitude Est ; $0^{\circ}$ et $5^{\circ}$ de latitude Nord, en climats sahélien et soudanien (la pluviométrie est supérieure à $600 \mathrm{~mm}$ ). La végétation est composée de forêts sèches basses, de forêt-galeries, de forêts claires, de savanes, de fourrés à Combretum et de steppes (Mahamane et al., 2009). La région de Dosso compte 2037713 habitants soit $49,35 \%$ d'hommes et $50,65 \%$ de femmes avec une densité de $60,2 \mathrm{hts} / \mathrm{km}^{2}$. Quant à la région de Tillabéri, elle compte 2722482 habitants soit $49,45 \%$ d'hommes et 
$50,55 \%$ de femmes avec $27,99 \mathrm{hts} / \mathrm{km}^{2}$ (INS, 2014). Dans toutes ces régions, l'agriculture et l'élevage constituent les principales activités socio-économiques de la population.

Collecte de données : Les données ont été collectées en suivant deux approches, une approche qualitative à travers de focus-group à l'aide d'un guide d'entretien permettant de cibler les personnes ayant des connaissances avérées sur l'espèce ou des personnes ressources désignées par les groupes. Puis une approche quantitative qui a consisté à des interviews sur la base d'une fiche de questionnaire adressé à chaque répondant, après son identification. La fiche est composée comme suit :

$>\quad$ le niveau de connaissances scientifiques indigènes sur l'espèce qui déterminent:

* les noms vernaculaires de l'espèce ;

* les différentes variétés présentes dans le terroir ;

* les types de sol où cette plante se développe ;

* le délai de sa première production fruitière production ;

$>$ les perceptions des populations locales de la dynamique des populations naturelles de $D$. mespiliformis:

* la situation des grandes zones de peuplement et son origine ;

* l'effet interactif de ce peuplement avec son environnement immédiat.

* l'état actuel des populations de la plante ;

* les stratégies locales de conservation;

$>$ les valeurs socioéconomiques et culturelles de l'espèce entre autres :

* la disponibilité des fruits et ses utilisations ;

* les fournisseurs de ces fruits ;

* les sources d'approvisionnement des fruits et le gain annuel ;

* les utilisations des différentes parties de l'espèce et ses effets ;

Les questionnaires ont été élaborés de manière à ce que certaines questions pour lesquelles les variables sont maîtrisées, soient fermées, alors que d'autres qui nécessitent un certain approfondissement, soient ouvertes. Chaque fiche de questionnaire a été administrée à un paysan, ce qui a permis d'acquérir auprès des populations locales des données statistiquement exploitables. L'échantillonnage a été stratifié en trois niveaux. Le premier niveau est la subdivision phytogéographique du Niger (Saâdou, 1990), le deuxième est le zonage hydrographique (réseaux hydrographiques du Niger) et le troisième est la division administrative (village). Les villages sont choisis selon la répartition géographique de $D$. mespiliformis. Les trois strates ont été prises en considération dès que possible pour interviewer individuellement, toute personne acquérant des connaissances avérées sur l'espèce, des liens avec les fonctions et services fournis par D. mespiliformis. Ainsi, pour tenir compte des zones de distribution de l'espèce, cinq compartiments phytogéographiques ont été retenus (Figure 1) à savoir :

\section{-Compartiment Nord-soudanien occidental} (A1)

La pluviométrie est supérieure à $600 \mathrm{~mm}$ et la végétation est composée de forêt sèche basse sur les plateaux latéritiques, forêt-galerie sur les berges des Dallols, forêt claire sur les terrasses argileuses méridionales et dans les toposéquences de vallées, savanes dans les vallées sèches et sur les dunes fixées surplombant les vallées.

\section{-Le compartiment Nord-Soudanien central} (A2)

La pluviométrie est supérieure à $600 \mathrm{~mm}$ et la végétation est composée de forêt sèche basse sur les plateaux, forêts-galeries sur les berges des cours d'eau, savanes sur les terrasses sableuses, les dunes et les vallées sèches.

\section{- Le compartiment Sud-Sahélien occidental} (B1)

La pluviométrie est comprise entre 400 et 600 mm et la végétation est composée de fourrés à Combretum sur les plateaux latéritiques, 
steppes sur les terrasses sableuses, dans les vallées sèches et sur les dunes fixées.

-Le compartiment Sud-sahélien central (B2)

La pluviométrie est la même que dans le compartiment précédent. La végétation est également composée de fourrés à Combretum sur les plateaux latéritiques mais avec des savanes sur les terrasses sableuses méridionales et steppes sur les dunes et dans les vallées sèches.

\section{-Le compartiment Nord-sahélien} occidental $(\mathbf{C l})$

La pluviométrie est comprise entre 200 et 400 $\mathrm{mm}$ et la végétation est composée de steppes arborées dans les zones basses et steppes arbustives sur les substrats sableux.

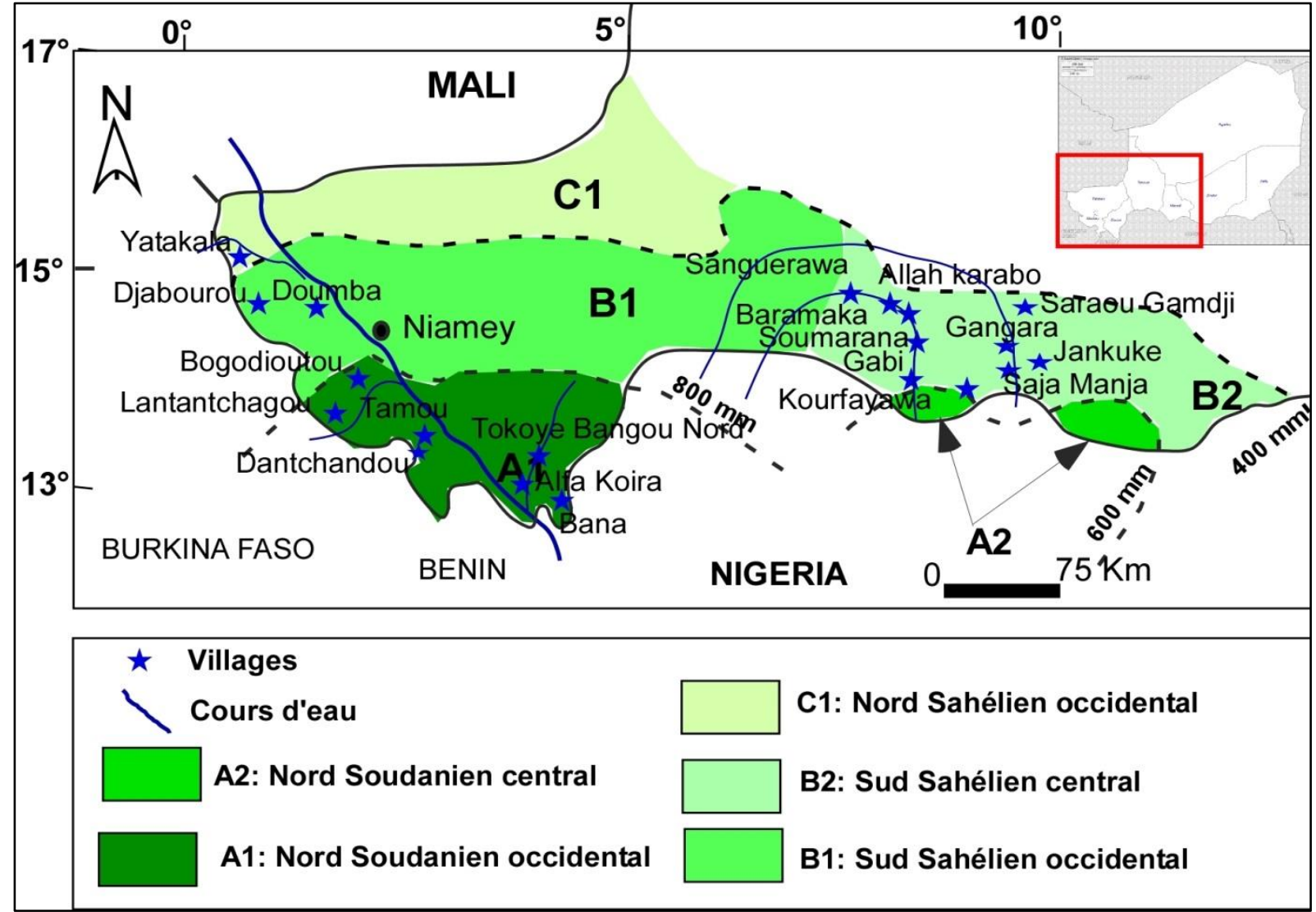

Figure 1 : Subdivision phytogéographique du Niger (Source : Saadou, 1990)

Dans chaque compartiment, des cours d'eau ont été retenus par hasard. Ainsi, le Dallol Foga, le Dargol, le Djiamangou, le Goroubi, le Goulbi Maradi et le Goulbi N'kaba ont été concernés. Un total de 20 villages admiratifs
(10 villages au centre et 10 villages à l'Ouest du Niger) ont été retenus et comptant 600 personnes en raison de 30 personnes par village dont la structure des répondants est répresentée dans le tableau 1. 
Tableau 1: Répartition des répondants

\begin{tabular}{l|l|l|l|l}
\hline Modalité & Adolescents & Jeunes & Vieux & Total \\
\hline Masculin & 3 & 62 & 237 & 302 \\
Féminin & 4 & 101 & 187 & 292 \\
Total & 7 & 169 & 424 & 600 \\
Fréquence (\%) & 1 & 28 & 71 & 100 \\
\hline
\end{tabular}

Analyse des données: Les données quantitatives d'enquête ont fait l'objet de dépouillement et de saisie aux moyens de logiciels appropriées de traitement de données. Le logiciel SPSS (version 20.0) a permis de mener les opérations suivantes : codification, la conception du masque de

- $\quad$ Fréquence de citations

Pour chaque catégorie d'usage, nous avons analysé la Fréquence de Citations (FC)

$\mathrm{FC}=\frac{\text { Nombre de citations } \mathrm{d}^{\prime} \text { une partie }}{\text { Nombre total de répondants }} \times 100$

- Valeur d'usage totale rapportée

La valeur d'usage totale rapportée (VUR) pour un groupe est le nombre total moyen d'usages spécifiques rapportés pour $D$.

$V U R=\frac{\sum_{i=1}^{N} V U R_{i}}{N}$

où VURi est le nombre total d'usages spécifiques rapportés par l'enquêté i du groupe ; $\mathrm{N}$ est le nombre total d'enquêtés pour le groupe social considéré. La valeur d'usage rapportée par partie/organe (VURorg)

$V U \operatorname{Rorg}_{x}=\frac{\sum_{i=1}^{N} V U R_{x i}}{N}$

où VURorgx est la valeur d'usage rapportée pour la partie/ organe x; VURxi est le nombre total d'usages spécifiques de la partie/organe $\mathrm{x}$ mentionné par l'enquêté $\mathrm{i}$ du groupe considéré ; $\mathrm{N}$ est le nombre total d'enquêtés du groupe. Cet indice a permis de mesurer et de comparer les connaissances des enquêtés entre les différents groupes socioculturels, catégories d'âges et sexes. Les tranches d'âge retenues pour les analyses statistiques sont : Adolescents âge compris entre [0_18 [, les jeunes d'âge compris entre [18_45 [, les vieux qui ont plus de 45 ans. Les groupes présentant des VUR élevées sont ceux possédant usages spécifiques par enquêté : enquêté : saisie, l'entrée des données, la tabulation et le tri à plat (tirage des fréquences simples). Excel a servi aussi à calculer les moyennes, les différents indices ethnobotaniques et à construire les graphiques. Des indices ethnobotaniques ont été calculés à travers les formules :

mespiliformis dans ce groupe, exprimé en

(Gomez-Beloz, 2002) est le nombre moyen d'usages rapportés pour chaque organe de $\mathrm{B}$. costatum, exprimé en usages spécifiques par

globalement plus de connaissances sur les usages de la plante. Il a également permis d'évaluer le nombre d'usages connus par partie/organe de la plante. Les parties/organes présentant des VURorg élevées sont ceux ayant le plus grand nombre d'usages, et donc probablement les plus souvent sollicités par les populations. Afin de déceler l'existence d'interaction entre la diversité des cultures et l'utilisation des organes/parties de $D$. mespiliformis, les Valeurs d'Usage des tous les organes/parties utilisés par les ethnies ont été soumises à une analyse en composante 
principale (ACP) à l'aide du logiciel Minitab.16.

\section{- Indice d'importance culturelle (IC)}

L'indice d'importance culturelle (IC) (Houehanou et al., 2011) désigne, d'une part, la valeur culturelle allouée à la plante et, d'autre part, celle rattachée aux parties/organes de la plante par les populations locales. La valeur de l'IC a été calculée par catégorie d'usages pour chaque groupe socioculturel d'une part et pour chaque partie/organe de la plante d'autre part. Dans le premier cas, l'IC permet de mettre en exergue la catégorie d'usages la plus importante selon les groupes socioculturels et, dans le second cas, la catégorie d'usages la plus importante selon la partie/organe de la plante. La valeur

$C I=\sum_{U=U_{1}}^{N C} \cdot \sum_{I=1}^{I_{N}} \frac{U R_{u i}}{N}$

$\mathrm{NC}$ : nombre total de catégories d'usages, UR : nombre d'usage rapporté de l'espèce et $\mathrm{N}$ : nombre total d'informateurs.

\section{- Facteur de Consensus Informateur}

(FCI) : Le niveau de consensus des populations sur les usages des ressources ligneuses a été appréhendé par le calcul du Facteur de Consensus Informateur (FCI) ou Informant Consensus Factor défini par Heinrich et al. (1998). Les valeurs du FCI sont $F C I=\frac{N u r-N t}{N u r-1}$

où Nur $=$ nombre de citations pour chaque catégorie, $\mathrm{Nt}=$ nombre d'espèces pour cette même catégorie.

- Niveau de Fidélité (NF) : En s'inspirant de l'utilisation du Niveau de de IC a été par ailleurs calculée par partie/organe et pour chaque groupe socioculturel afin de mettre en exergue les parties/organes de plantes les plus importants pour chaque groupe socioculturel. Selon Tardio \& Pardo-de-Santayana (2008), cet indice serait plus approprié pour mesurer la variation de la connaissance entre différentes communautés. Ces données ont été soumises à une Analyse Factorielle de correspondance (AFC) pour déterminer les ressemblances les groupes homogènes selon les facteurs déterminants identifiés par les différentes groupes (ethnies, âges et les sexes). L'analyses a été réalisée dans le logiciel Minitab 16.0. Pour chaque groupe ethnique l'indice d'importance culturelle a été calculé en utilisant la formule ci-dessous :

comprises entre 0 et 1 . Une valeur élevée de FCI (plus proche de 1) est obtenue quand un seule ou un nombre réduit d'espèces est cité par une grande proportion d'informateurs pour une catégorie de service spécifique. À l'inverse, sa valeur sera d'autant plus faible (plus proche de 0) quand une grande diversité d'espèces citées pour un même usage. Le FCI est calculé par la formule suivante :

Fidélité en ethnomédecine (Ugulu I, 2012), nous avons défini le Niveau de Fidélité (NF) d'une espèce par rapport à différentes catégories d'usages.

$\mathrm{NF}=\quad \frac{\text { Nombre de citaions de partie pour une cartegorie }}{\text { Nombre de citation de partie pour toutes les categories }}$

En ce qui concerne les informations qualitatives, il a été question de réaliser un recoupement des différents points de vue issus des entretiens et observations. Leur confrontation avec les informations quantitatives a permis de renforcer les analyses. Les données collectées ont été traitées avec le logiciel SPSS.Statistics.20. Une analyse en Composante principale (ACP) a été réalisée pour déterminer les ressemblances et les groupes homogènes selon les facteurs déterminants identifiés par les différentes ethnies. 


\section{RÉSULTATS ET DISCUSSION}

Niveau de Connaissance de $D$. mespiliformis : Toutes les personnes interviewées connaissent $D$. mespiliformis. Ainsi, selon les langues, on l'appelle adotan (tamacheq), gaanaahi ou pupuy (peuhl), kaiwa ou kanya (haoussa), tokoey nya (zarma) doukouhi (gourmantché). Les variétés se différencient par la taille de leurs fruits et surtout la période de leurs maturités. Il y a des variétés hâtives qui murissent en décembre et des variétés tardives «maiwa» qui même après maturation ne sont forcément pas jaunes et qui peuvent rester jusqu'aux mois de Juin et Juillet. Selon les paysans, cette espèce se trouve uniquement au bord des cours d'eau et surtout sur le sol argileux à l'Ouest du Niger alors qu'elle est présente dans les champs éloignés des cours d'eau sur des sols sableux et limoneux au centre du Niger. Les variétés les plus prisées sont surtout les plus sucrées, charnues et/ou les plus grosses et renfermant peu ou pas de graines. Environ quarante-six (46) usages de l'espèce ont été notés chez les différents groupes socioculturels et socioprofessionnels. Ils ont été regroupés dans
8 principales catégories d'usages (tableau 1). En termes d'utilisations médicinales, les feuilles, la racine, l'écorce et la sève sont beaucoup utilisées dans le traitement de plusieurs maladies (tableau 2). Près de 33 maux sont guéris par les organes du $D$. mespiliformis selon les répondants. L'analyse de ce tableau montre par ailleurs que le bois de D. mespiliformis est beaucoup utilisé comme bois d'énergie, bois de service et bois d'œuvre. Il est aussi vendu sur pied ou sous forme de grume, charbon et de meubles. Cette diversité d'utilisation est étroitement liée à sa composition biochimique (Charles et al:, 2009 ; Ali et al., 2012 ; Shagal et al :, 2012 ; Ebbo et al., 201; Mahudro Yovo et al :, 2020). Ainsi les travaux de Ebbo et al en 2020 ont montrés que l'extrait méthanolique brut des parties végétales de $D$. mespiliformis a une DL50 $\geq 5 \mathrm{~g} / \mathrm{kg}$ après administration orale chez le rat, donc sans danger selon les normes de l'OCDE. Dans les études de toxicité subchronique, les fractions d'acétate d'éthyle des feuilles et de l'écorce de la plante étaient relativement sûres $(\mathrm{P}>0,05)$. 
Tableau 1: Catégorie d'usage de Diospyros mespiliformis

\begin{tabular}{|c|c|c|}
\hline $\begin{array}{l}\text { Catégorie } \\
\text { d'usage }\end{array}$ & organe/partie & usages spécifiques \\
\hline Alimentaires & \begin{tabular}{|l|} 
Feuille \\
Fleur \\
Fruit \\
\end{tabular} & \begin{tabular}{|l|} 
salade, ingrédient de sauce \\
mélangé avec la jeune feuille \\
consommé cru à maturité
\end{tabular} \\
\hline Artisanale & $\begin{array}{l}\text { Bois } \\
\text { Racine }\end{array}$ & $\begin{array}{l}\text { jougs, mortier, pilon, manches de matériels agricoles } \\
\text { matériels aratoires }\end{array}$ \\
\hline Bois de chauffe & Bois & Charbon \\
\hline Commerce & Fruit & en gros ou en détaille \\
\hline Construction & Bois & case, hangar, clôture \\
\hline $\begin{array}{l}\text { Culte-médico- } \\
\text { magie }\end{array}$ & Racine & chasse les mauvais esprits \\
\hline Fourrage & Feuille & sur pied ou, apporté aux animaux attachés \\
\hline médicinale & $\begin{array}{l}\text { Ecorce } \\
\text { Feuille } \\
\text { Fleur } \\
\text { Fruit } \\
\\
\text { Racine }\end{array}$ & $\begin{array}{l}\text { cicatrisation, hémorroïde, maux de ventre, reflux } \\
\text { gastrique, maladies cutanées, épilepsie, rhumatisme } \\
\text { osseux, tuberculose osseuse, céphalée, ulcère, maux de } \\
\text { cœur, dysménorrhée } \\
\text { antipaludéen, rhumatisme enfantin, antivenimeux, } \\
\text { constipation, diarrhée, vermifuge } \\
\text { Anti-diarrhée, ulcère } \\
\text { maux de ventre, anti-acier } \\
\text { fièvre jaune, cancer de sein, syphilis, antivenimeuse, } \\
\text { antifatigue, anti-infectieux, cicatrisation de brûlure, } \\
\text { aphrodisiaque }\end{array}$ \\
\hline
\end{tabular}

La première production de l'espèce constitue un défi pour les paysans. Seulement 45,5\% ont estimé l'âge de sa première production fruitière (Figure 2). Cet âge varie selon les zones écologiques, il est de 5 à 11 ans selon
$83,32 \%$ des enquêtés et seuls $4,17 \%$ pensent que ce délai peut atteindre 20 ans. En plus, le délai de la première production varie suivant le gradient pluviométrique. Il est plus court dans les zones les plus arrosées. 


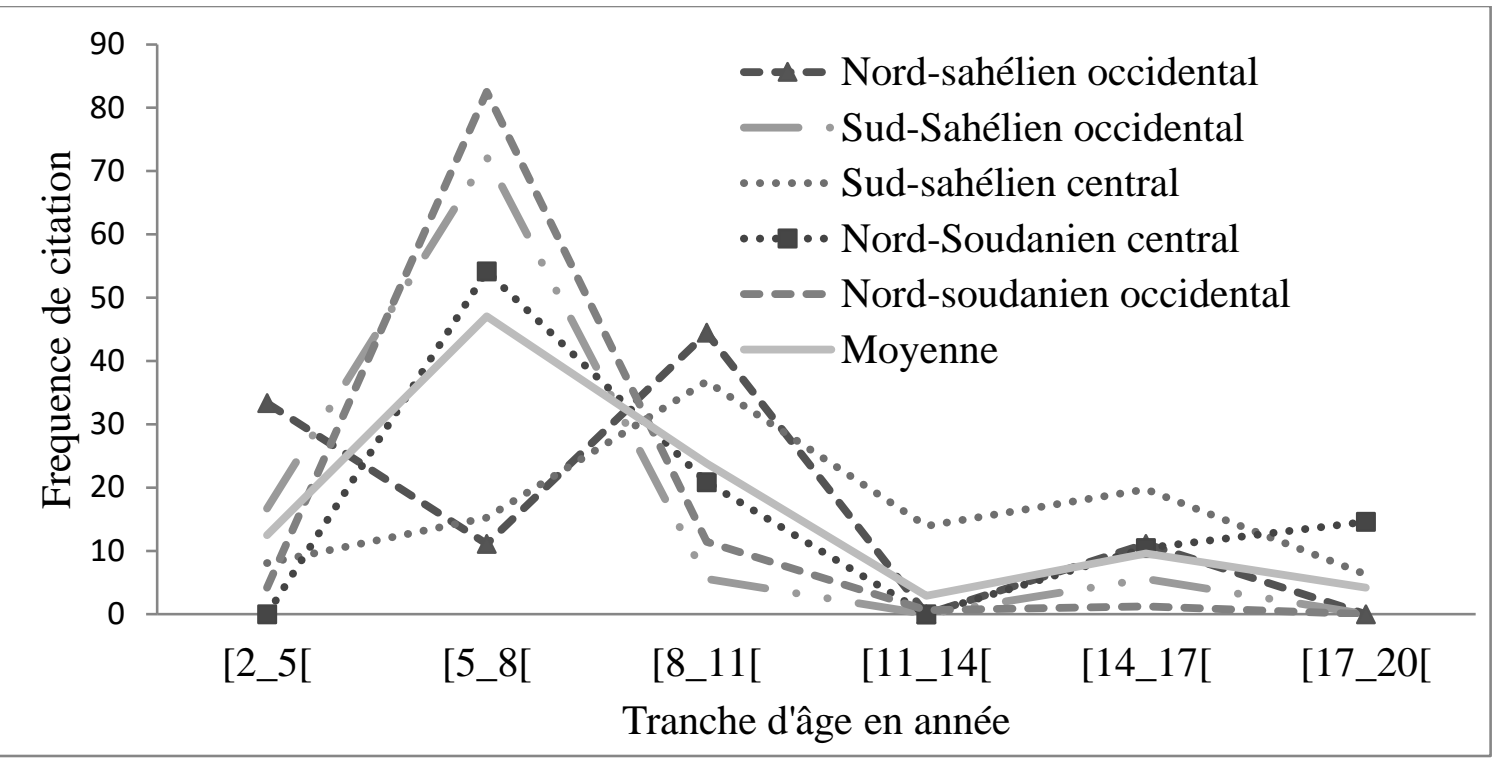

Figure 2 : Répartition de l'âge de la première fructification de plant de D. mespiliformis

Perceptions des populations locales de la dynamique des populations naturelles de D. mespiliformis : D. mespiliformis est issu d'un peuplement naturel c'est-à-dire elle se trouve à l'état sauvage. Toutes les catégories de répondants sont conscientes de la régression des populations de l'espèce dans l'ensemble des sites avec une proportion plus importante dans le centre du pays. Les raisons de cette régression sont essentiellement d'ordre anthropique (la coupe abusive) consécutive à l'augmentation de la population humaine et qui a pour conséquence l'occupation de terre à des fins agricoles au détriment des ressources végétales dont $D$. mespiliformis. Ce phénomène est accentué par la baisse et ou l'irrégularité de la pluviométrie. A cela s'ajoute, selon les populations rurales, le non renouvellement des populations de l'espèce qui est la conséquence directe de la longue saison sèche et une sécheresse récurrente qui ont des effets négatifs sur les jeunes sujets (Ali et al., 2017). Les ruissellements participent aussi à la destruction de l'espèce surtout les gros sujets vieillissants se trouvant sur les lits des cours d'eau. Néanmoins, $47 \%$ de répondants pensent qu'en milieu naturel des jeunes semis et rejets de très petites tailles sont présents.
Les paysans sont conscients que l'espèce ne fait pas l'objet d'une protection particulière à leur niveau et au niveau du service des eaux et forêts comme certaines espèces telle que Faidherbia albida car au Niger il existe 14 espèces ligneuses protégées (Saadou, 2007). Cependant, il existe quelques rares cas où les pieds se trouvent dans les champs ou les jardins et par conséquent ils sont les propriétés exclusives du propriétaire terrien.

Valeurs socioéconomiques et culturelles de l'espèce: L'analyse en composante principale révèle que les composantes une et deux contribuent respectivement $70,6 \%$ et $13,9 \%$ de l'explication de la variabilité observée (Figure 3). Les deux premiers axes expliquent $84,5 \%$ de la variabilité des maladies traitées par les organes de D. mespiliformis en fonction des ethnies au Niger. Ainsi, la diversité socioculturelle se manifeste dans l'utilisation de la même ressource. Les résultats indiquent l'existence de liens étroits entre les groupes et l'usage de $D$. mespiliformis. Les résultats indiquent que, les vieux et de sexe masculin se distinguent par d'importantes connaissances des vertus $D$. mespiliformis. La plupart d'adolescents ne connaissent que l'utilisation de Bois en énergie, l'écorce et racine en pharmacopée 
traditionnelle. Cela peut être expliqué par le fait que l'essentiel de la connaissance en Afrique est transmise par l'orale et que cette transmission exige une certaine maturité d'esprit et surtout un certain état de confiance entre les apprenants les détenteurs du savoir qui sont pour la plupart des cas des vieux. Des résultats similaires ont été obtenus sur l'utilisation des espèces ligneuses telles Pterocarpus erinaceus au Togo (Kossi et al., 2016) et Burkina et Niger (Habou, 2017).

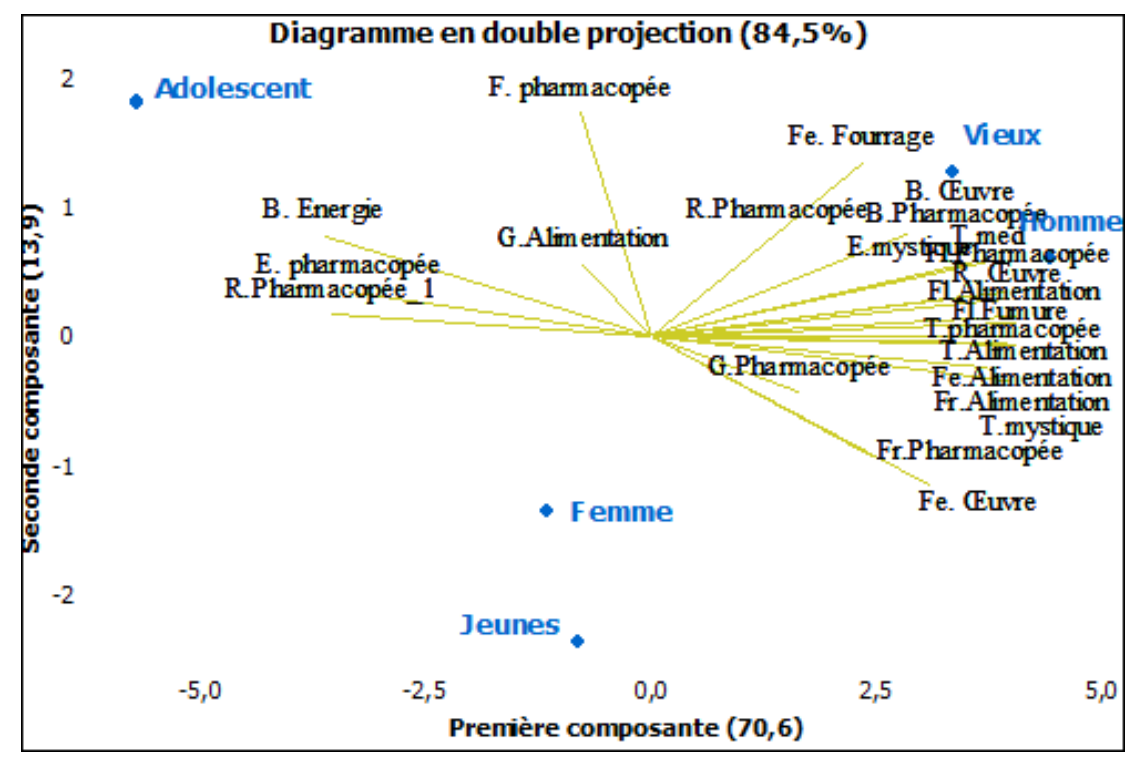

Figure 3: Relation entre les différents usages de D. mespiliformis et les groupes ethniques des enquêtés.

(Avec $\mathrm{B}=$ bois, $\mathrm{E}=$ =écorce, $\mathrm{Fe}=$ feuille, $\mathrm{Fl}=$ Fleur, $\mathrm{Fr}=$ fruit, $\mathrm{G}=$ gui, $\mathrm{Gr}=$ graine, $\mathrm{R}=$ racine).

Les données sur les fréquences des citations des produits issus de $D$. mespiliformis et les compartiments phytogéographiques ont été soumises à l'analyse factorielle des correspondances (AFC) qui démontre que les deux premiers axes concentrent plus de $89,58 \%$ de la variance totale. L'axe 1 centralise à lui seul 67,36\% et le deuxième axe 22,22\% (Figure 4). L'analyse du plan factoriel montre que les modalités Nord-Soudanien central et Nord-sahélien occidental contribuent le plus respectivement à la construction de l'axe 1 et de l'axe 2. Au niveau des utilisations, c'est celles des Fleurs et des graines qui sont plus corrélées avec ces deux axes tandis qu'il ressort une faible corrélation d'une part entre les autres parties/organes et d'autre part entres les compartiments phytogéographiques. Cela constitue un indicateur au fait que l'utilisation est plutôt culturelle que la fonction la répartition spatiale de la population car la population du pays connait un grand mixage culturelle avec les mariages interethniques. 


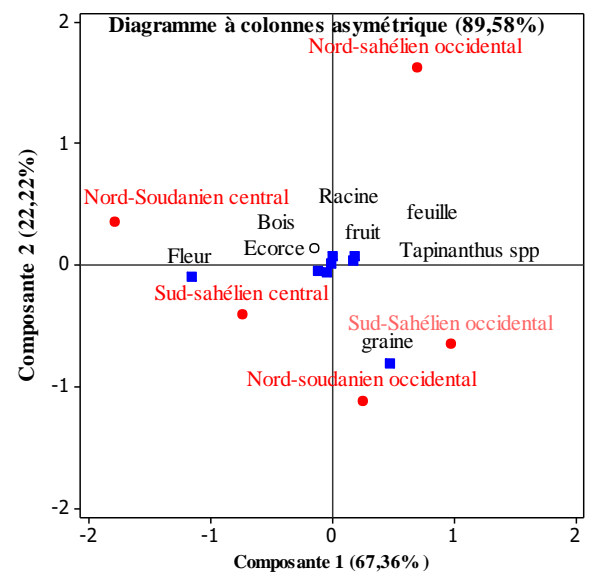

Figure 4: Relation entre les fréquences des citations des parties de D. mespiliformis et les différents sites.

D. mespiliformis produit des fruits qui sont utilisés pour la consommation humaine, surtout en période de maturité qui dure de 3 à 5 mois. Mieux encore, $30 \%$ des personnes enquêtées indiquent que les fruits sont conservés pendant toute l'année. Les fruits sur les pieds mères ne durent que trois mois. Pour la consommation des fruits de l'espèce, $10 \%$ des personnes enquêtées achètent les fruits avec les enfants, $8 \%$ avec les femmes et $15 \%$ les femmes et les enfants et le prix varie entre 50 FCFA et 600 FCFA la tiya $(1,5 \mathrm{~kg})$ dans les villages mais certainement plus chère en milieu urbain. La vente procure aux concernés un revenu annuel de 5000 à 27000 FCFA. En marge de $D$. mespiliformis d'autres espèces fruitières sont consommées dont les plus importantes sont Ziziphus mauritiana Lam., Balanites aegyptiaca (L.) Del., Lannea microcarpa Engl. \& K. Krause, Parkia biglobosa (Jacq.) R. Br. ex G. Don, Tamarindus indica L. L'ensemble de personnes interviewées rapportent que la production fruitière varie d'une année à l'autre, dont les principales raisons sont la pluie, la température et le vent. Concernant l'utilisation des autres parties de l'espèce, presque toutes les parties sont utilisées par la population locale. La figures $5 \mathrm{~A}$ montre les valeurs d'usage des différents organes/parties de $D$. mespiliformis. l'analyse de cette figure révèle que l'écorce constitue la partie la plus utilisée par la population rurales et la fleur est moins utilisée. La valeur d'usage rapportée varie dans le même sens que le niveau de fidélité tandis que le facteur de consensus d'informateur proche de 1 pour toutes les catégories d'utilisation (Figure 5). La pharmacopée traditionnelle constitue la première utilisation de $D$. mespiliformis au Niger suivit de l'utilisation alimentaire, le bois-énergie et bois d'œuvre. Les résultats ont montré que $D$. mespiliformis est une espèce très appréciée et beaucoup plus utilisée en pharmacopée traditionnelle. Les paysans ont développé des mythes autour d'elle. Les connaissances indigènes acquises traduisent une étroite relation entre les paysans et cet arbre est considéré comme magique, surtout chez les Sorko «pécheurs» qui 1'utilisent pour attirer les poissons qui, semble-t-il, ne voient pas les filets et par conséquent sont piégés et attrapés. Le bois et les racines sont utilisés par les paysans non seulement pour les travaux ménagers mais aussi comme source d'énergie. Deux modes d'approvisionnement sont à distinguer : l'auto-approvisionnement et la filière commerciale (FAO, 1999). L'autoapprovisionnement concerne la majeure partie du bois consommé dans le pays, soit 2,4 millions de tonnes en 2000 (FAO, 1999). Yaméogo et al., (2013) ont montré que le bois de cette espèce est aussi utilisé au Burkina Faso. Les fruits sont très bien utilisés en alimentation humaines et ils constituent un important apport nutritionnel. En effet, les 
travaux de Chivandi et al (2009) ont montré que les fruits contiennent des acides gras $(0,7 \%)$ dont $\mathrm{C} 14: 0$ (Acide myristique) $0,62 \%$; C16:0 (Acide palmitique) 30,06\%; C18:0 (Acide stéarique) 7,74 \%; C20:0 (Acide arachidique) $1,12 \%$ qui constituent l'essentiel des acides gras athérogènes dont le besoin est estimé à $8 \mathrm{~g} / \mathrm{j}$ contre $12 \mathrm{~g} / \mathrm{j}$ (Recommandations nutritionnelles (2010) la somme des trois acides gras athérogènes est fixée à $8 \mathrm{~g} / \mathrm{j}$ contre $12 \mathrm{~g}$ pour l'ensemble des acides gras). Ces mêmes auteurs ont montré que l'huile de fruits, en raison des son niveau élevé, était précieuse en tant que complément alimentaire,
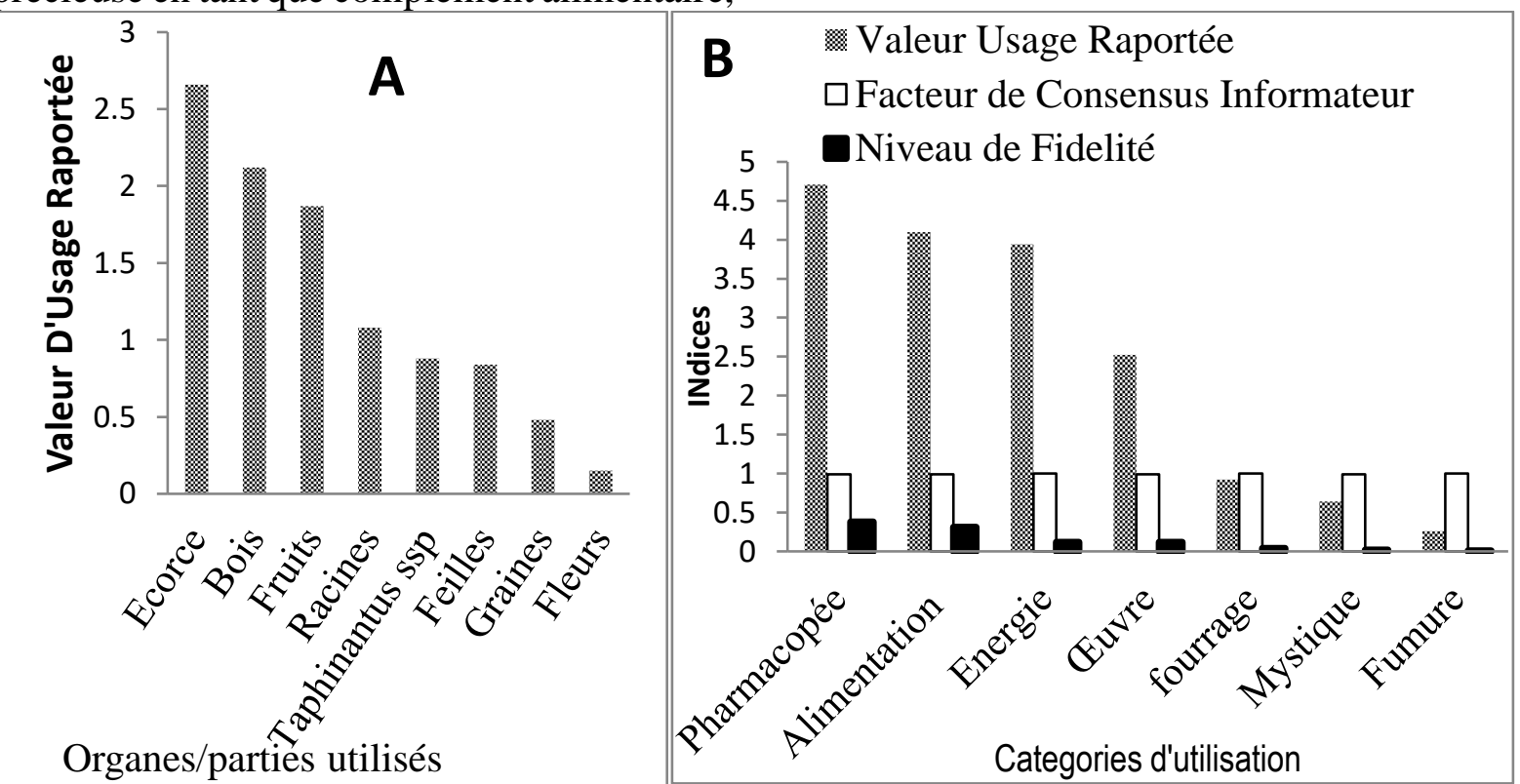

Figure 5 : Indices ethnobotaniques A : Valeur d'Usage Rapportée par organes/parties et B : Valeur d'Usage

Rapportée par catégories d'utilisation, Facteur de Consensus Informateur et Niveau de Fidélité : L'analyse factorielle des correspondances effectuée pour décrire la relation entre les catégories d'usages des organes/parties et les différentes ethnies considérées montre que les deux premiers axes expliquent $71,55 \%$ de la variabilité observée (Figure 6). Ces deux premiers axes (F1 et F2) concentrent respectivement 44,97\% et $26,58 \%$ de l'information. Les modalités Usages Energie et Fourrage contribuent le plus respectivement à la construction de l'axe pour la synthèse des icosanoïdes et aiderait à atténuer le dysfonctionnement des systèmes cardiovasculaire, rénal, reproductif, gastrointestinal et immunitaire. Mieux, l'analyse des fruits au Nigeria (Ebbo et al., 2020) a montré que la composition des fruits par $100 \mathrm{~g}$ de portion comestible est la suivante : eau :69 g, énergie : $404 \mathrm{~kJ}$ (97 kcal), protéines : 1,1 g, lipides : 0,4 g, glucides : $22 \mathrm{~g}$, fibres : $6,2 \mathrm{~g}$, $\mathrm{Ca}: 96 \mathrm{mg}, \mathrm{Mg}: 28 \mathrm{mg}, \mathrm{P}: 46 \mathrm{mg}, \mathrm{Fe}: 1 \mathrm{mg}$, thiamine : $0,01 \mathrm{mg}$, riboflavine : $0,04 \mathrm{mg}$, niacine : 0,24 $\mathrm{mg}$ et acide ascorbique 24,6 : $\mathrm{mg}$ 


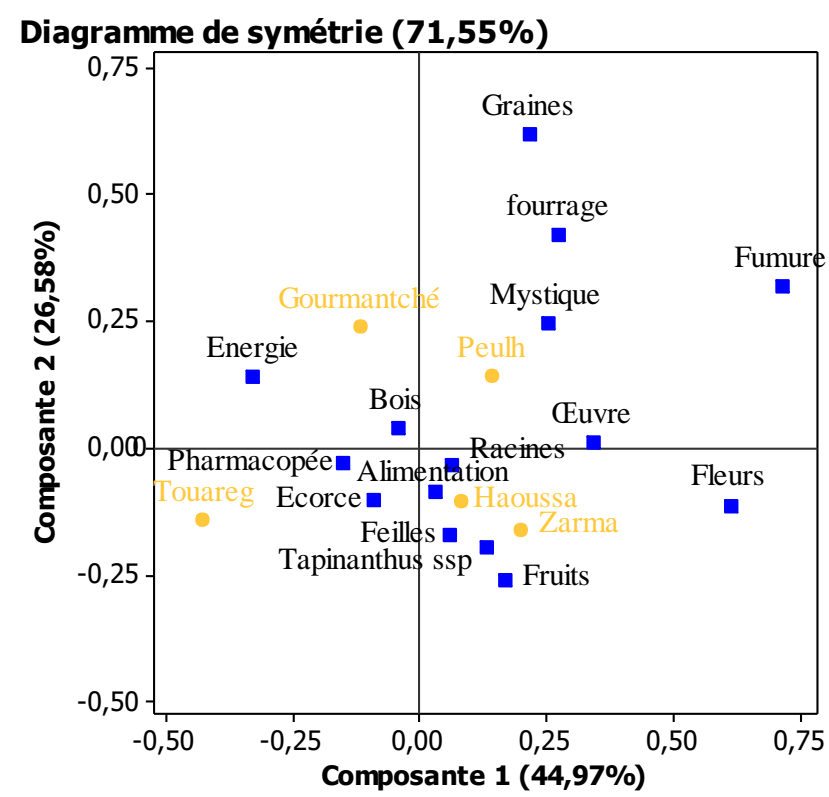

Figure 6 : Plan factoriel décrivant la relation entre les ethnies et les domaines d'utilisation de l'espèce

\section{CONCLUSION}

A l'issue de cette étude, il ressort que la population rurale connaît bien $D$. mespiliformis et qu'elle lui rend des fonctions et services importants même si elle ne fait pas l'objet de plantation. Les usages de $D$. mespiliformis sont relatifs aux groupes ethniques. C'est une espèce qu'on rencontre dans les sols hydromorphes (surtout argileux, sableux et limoneux). Elle fait partie intégrante de leurs activités quotidiennes. Elle est considérée comme un arbre à pouvoir mystique car son tronc noir fait d'elle un remède contre tous les mauvais esprits qui ont peur du noir. Les enquêtés sont unanimes sur l'utilisation des fruits de l'espèce dans

\section{RÉFÉRENCE BIBLIOGRAPHIQUE}

Akpona T. J. D.,Glèlè Kakaï R. L. and Akpona A. H. 2009. Ethnobotanique et structure des peuplements d'espèces de brosse végétale au Sud et au Centre du Bénin. Bulletin de la Recherche Agronomique du Bénin. Pp 1-12. l'alimentation humaine. Les autres parties (écorce, feuille, fleurs, fruits, graines et racine) connaissent beaucoup plus des utilisations pharmaceutiques. Ces utilisations ne sont pas sans conséquence sur la survie de l'espèce même si la réduction de la population de l'espèce est surtout causée par les phénomènes naturels (sécheresses et vieillissement) accompagnés des actions anthropiques. Aucune disposition particulière n'est prise jusque-là pour réduire l'érosion génétique de cette essence forestière et pourtant cela s'avère nécessaire compte tenu de différentes menaces.

Ali A, Abdou L, Douma S, Mahamane A, Saadou M, 2016. Les ligneux alimentaires de soudure dans les communes rurales de Tamou et Tondikiwindi: diversité et structure des populations. Journal of Animal \& Plant Sciences. 31(1) pp 4889-4900. 
Ali A, Boubé M, Maman M I, Salamatou A, Ali $M$ et Mahamane S. 2017. Caractérisation des peuplements ligneux des parcs agroforestiers à Diospyros mespiliformis dans le centre du Niger. Afrique SCIENCE 13(2) pp 87 - 100.

Ali A. J, Usman. T, Abdulrazaq.T, Rukkaya. S. S and Patience. S. K. (2012). Effects of Sub-Chronic Administration of Diospyros Mespiliformis Hochst (Ebenaceae) Root Extracts on Some Biochemical Parameters in Mice; Journal of Applied Pharmaceutical Science, $\quad 02 \quad(05) ; \quad 60-64$. https://doi.org/10.7324/JAPS.2012.25 $\underline{08}$

Arbonnier M, 2000. Arbres, arbustes et lianes des zones sèches d'Afrique de l'Ouest. CIRAD - MNHN - UICN, Montpellier (France), $541 \mathrm{p}$.

Charles O.E., Chukwuemeka S N., Ebere B O., Justina U O., Kelechi L N., Joy C O., Maria I N., Kennedy F.C. (2009). Anti-mycobacterial activity of root and leaf extracts of Anthocleista djalonensis (Loganiaceae) and Diospyros mespiliformis (Ebenaceae). Chivandi.E, Eriwanger. KH, Davidson. BC. (2009). Lipid content and fatty acid profile of the fruit seeds of Diospyros mespiliformis. International Journal of Integrative Biology 5; 121-124

Ebbo AA, Mammam M, Suleiman MM, Ahmed A and Bello A. 2014. Preliminary Phytochemical Screening of Diospyros Mespiliformis. Anat Physiol 4:4. https://doi.org/10.4172/2161$\underline{0940.1000156}$

Ebbo AA, Sani D, Suleiman MM, Ahmad A, Hassan AZ. (2020); Acute and subchronic toxicity evaluation of the crude methanolic extract of Diospyros mespiliformis hochst ex a. Dc (ebenaceae) and its fractions.
Toxicology Reports 7 pp 1138-1144. https://doi.org/10.1016/j.toxrep.2020. 08.028.

PMid:32983901

PMCid:PMC7493130

Fandohan B, KAKAI RG, SINSIN B, PELZ D. (2008). Caractérisation dendrométrique et spatiale de trois essences ligneuses médicinales dans la forêt classée de Wari-maro au Benin. Rev. Ivoir. Sci. Technol., 12. pp 173 186

FAO. 1999. Deuxième rapport sur l'état des ressources phytogénétiques pour l'alimentation et l'agriculture au Niger. P 68.

Habou R. 2017. Caractérisation des peuplements naturels de Pterocarpus erinaceus Poir. et élaboration de normes de gestion durable au Niger et au Burkina Faso (Afrique de l'Ouest).Thèse Doctorat unique Université de Niamey. 207 p.

Gome-Belo A. 2002. Plants use Knowlodge of the wikina Warao: The case for questionnaire in Ethnobotany. $\begin{array}{lll}\text { Economy } & \text { Botany.56 } & \text { (3) } 231 .\end{array}$ https://doi.org/10.1663/00130001(2002)056[0231:PUKOTW]2.0. $\mathrm{CO} ; 2$

Heinrich M, Ankli A, Frei B, Weimann C, and O. Sticher. 1998. Medicinal Plants in Mexico: Healers' Consensus and Cultural Importance. Social Science and Medicine 47:1859-1871. https://doi.org/10.1016/S02779536(98)00181-6

Houehanou TD, Assogbadjo AE, Glèlè Kakaï R, Houinato M, Sinsin B. (2011), Valuation of local preferred uses and traditional ecological knowledge in relation to three multipurpose tree species in Benin (West Africa). Forest Policy and Economics, 13 pp. 554562.https://doi.org/10.1016/j.forpol.2 $\underline{011.05 .013}$ 
Hutchings, A.H. Scott, G. Lewis, A. Cunningham Zulu Medicinal Plants Natal University Press, Pietermaritzburg (1996)

INS. (2014). Recensement général de la population et de l'habitat, 2012. Répertoire national des localités. Institut National de la Statistique, Niger.

Mahamane A, Mahamane S, Mohamed B D, Karim S, Bakasso Y, Abdoulaye D, Boubé M, Inoussa M M, Idrissa S \& Arzika T. 2009. Biodiversité végétale au Niger : état des connaissances actuelles. Ann. Univ. Lomé (Togo), Sciences, $18: 81-93$.

Saadou M., 1990. La végétation des milieux drainés nigériens à l'Est du fleuve Niger. Thèse de Docteur ès - Sciences Naturelles. - Université de Niamey. 395 p. annexes.

Saadou M., 2009. Quatrième Rapport National sur la Diversité Biologique (Niger). 95p.

Shagal M H, Kubmarawa D, and Alim H. 2012. Preliminary phytochemical investigation and antimicrobial evaluation of roots, stem-bark and leaves extracts of Diospyros mespiliformis. International Research Journal of Biochemistry and Bioinformatics .2(1) pp.011-015.

Sinsin B, Houinato M, Bruckmann A, ElHadj Issa A, Yaoitcha AS, Ballo MC, Ahokpè E, Mama A. 2004. Etude sur la biomasse dans la Réserve de Biosphère Transfrontalière du $\mathrm{W}$ "gestion des écosystèmes par le feu"

Tardío J, E. Blanco, A. M. Carvalho, J. J. Lastra, E. San Miguel, and R. Morales. 2007. Traditional Knowledge on Wild Edible Plants in the Northwest of the Iberian Peninsula (Spain and Portugal): A Comparative Study. Journal of Ethnobiology and Ethnomedicine 3:27. https://doi.org/10.1186/1746-4269-3$27 . \quad$ PMid:17555572 PMCid:PMC1904191

Ugulu I, 12.Fidelity Level and knowledge of Meical plants Used to Make Thepeutic Turkish Bath.Ethno Med, 6 (1).pp 1-9. https://doi.org/10.1080/09735070.201 2.11886413

Yaméogo G, Yélémou B, Kaboré O et Traoré D. 2013. Bois d'énergie du 'dolo' et bois de service : deux modes de consommation de bois à Vipalogo au Burkina Faso. Bulletin de la Recherche Agronomique du Bénin (BRAB). N73.p12.

Yovo M, Sedjro LOD, Philippe, Guy AA, Fidèle PT, Félicien A, Dominique CKS. 2020. Phytochimical studies and biological activities of extracts from two medical plants used in Benin to treat skin infections and septicemies.International journal of innovation and applied studies, 20(2), pp 507-514. 